\title{
SOME SHAPE OPTIMIZATION PROBLEMS FOR THE EIGENVALUES
}

\author{
YUSIF S. GASIMOV *
}

\begin{abstract}
In the work we consider some inverse problems relatively domain for the Laplace operator. The aim of the work is to find a domain under the condition that, the function, expressed by spectral data of the problem is given in the boundary of this domain.
\end{abstract}

Key words: Inverse problems, Eigenvalue, Eigenfunction, Convex domain, Support function.

AMS Classification Numbers: 49J45, 49Q10, 49R50

Introduction. Solution of a wide class of practical problems is reduced to the minimization of the functionals related with eigenvalues [1]. The study of shape optimization problems for the eigenvalues of an elliptic operator is a fascinating field that has strong relations with several applications as for instance the stability of vibrating bodies, the propagation of waves in composite media, the thermic insulation of conductors. Investigation of such problems is important also for study of the qualitative properties of the eigenvalues [2]. In the work we consider some inverse problems relatively domain. In its mathematical formulation the problem consists in taking an elliptic operator and considering its eigenvalues $\lambda_{k}$ as a functionals of the domain $D$ where the problem is solved. In the work these problems are reduced to the minimization of the functionals including spectral data of the corresponding operators. Usually in such problems obtained optimality conditions for domain involve eigenfunctions that makes difficult their use in the determination of the domain. Here we reduce the considering shape optimization problems to variational formulation, get some optimality conditions and a formula for the eigenvalue corresponding to the optimal domain. Note that the obtained formulas don't include an eigenfunction. It makes them interesting both from practical and theoretical points of view. For the sake of simplicity we consider only Laplace operator, but the results may be extended for other elliptic operators.

Main results. Consider the problem

$$
\begin{gathered}
-\Delta u=\lambda u, \quad x \in D, \\
u(x)=0, \quad x \in S_{D},
\end{gathered}
$$

where $\Delta$ - Laplace operator, $D \subset E^{n}$-convex bounded domain, $S_{D^{-i t s}}$ boundary, $\nabla=\sum_{i=1}^{n} \frac{\partial}{\partial x_{i}}$. It is known [3] that in the considered case the eigenfunctions $u_{j}, j=1,2, \ldots$ of the problem (1), (2) belong to the class $C^{1}(D) \cap C^{2}(\bar{D})$, eigenvalues are positive and may be numbered as $\lambda_{1} \leq \lambda_{2} \leq \ldots$, where each $\lambda_{k}$ is counted with its multiplicity.

Let $D \subset E^{n}$ be bounded convex domain with smooth boundary $S_{D}$. The set of all such domains we denote by $K$.

For the sake of simplicity we denote by $u$ the first normalized eigenfunction of the problem (1), (2) corresponding to the first eigenvalue $\lambda_{1}$.

The first problem is: To find a domain $D \in K$ such that for the first eigenvalue and corresponding eigenfunction of the problem (1)-(2) the relation

*Institute of Applied mathematics Baku State University, Z. Khalilov 23, AZ1148 Baku, Azerbaijan, e-mail: ysfgasimov@yahoo.com . 


$$
\frac{|\nabla u|^{2}}{\lambda_{1}}=\varphi(x), \quad x \in S_{D}
$$

is fulfilled for given in $E^{n}$ function $\varphi(x)$.

We call this problem $(\mathcal{I})$ problem.

As the seeking object of this problem is a domain its direct solution meets some difficulties. So, instead of this problem we consider the following one

$$
\begin{gathered}
-\Delta u=\lambda u, x \in D, \\
u(x)=0, \quad x \in S_{D}, \\
\lambda_{1}(D) \rightarrow \min ,
\end{gathered}
$$

under the condition

$$
\int_{D} f(x) d x=1
$$

where $f(x)$ is some given in $E^{n}$ function.

This problem we call $(\mathcal{V})$ problem.

Our aim is to show that for some classes of function $\varphi(x)$ the problems $(\mathcal{I})$ and $(\mathcal{V})$ are equivalent and to define these classes.

Let us introduce Lagrange's function

$$
L(D, \mu)=\lambda_{1}(D)+\mu \cdot \int_{D} f(x) d x .
$$

It is known that [4] for fixed domain $D$ the first eigenvalue of the problem (1), (2) is calculated by formula

$$
\lambda_{1}(D)=\inf _{u} I(u, D)
$$

where

$$
I(u, D)=\frac{\int_{D} \nabla u^{2}(x) d x}{\int_{D} u^{2}(x) d x}, \quad|\nabla u(x)|^{2}=\sum_{i=1}^{n}\left(\frac{\partial u}{\partial x_{i}}\right)^{2}
$$

and inf is taken over all functions $u \in E^{2}(D) \cap E^{1}(\bar{D})$, being equal to zero at $S_{D}$.

As we see, this formula defines $\lambda_{1}$ as a functional of $D$.

In $[5,6]$ it is proved that the first eigenvalue of the problem (1), (2) is differentiable with respect to $D$ on $K$ and for its first variation the formulae

$$
\delta \lambda_{1}(D)=-\int_{S_{D}}|\nabla u|^{2} \delta P_{D}(n(x)) d s
$$

is true.

Here $P_{D}(x)=\max _{l \in D}(l, x), x \in E^{n}$ is a support function of the domain $D, n(x)$ - outward normal to $S_{D}$ in the point $x$.

Denote 


$$
G(D) \equiv \int_{D} f(x) d x
$$

There is shown that this functional is also differentiable on $K$ and

$$
\delta G(D)=\int_{S_{D}} f(x) \delta P_{D}(n(x)) d s .
$$

Considering these formulas in (8) we get

$$
\delta L(D)=\int_{S_{D}}\left[\mu f(x)-|\nabla u|^{2}\right] \delta P_{D}(n(x)) d s .
$$

If $D$ is a solution of the problem (4)-(7) then as follows from Lagrange's theory the equality

$$
\mu \cdot f(x)-|\nabla u|^{2}=0, x \in S_{D}
$$

is fulfilled.

In spite of this condition is obtained under the convexity condition on $D$ one may show that it is true for non convex domains too.

Multiplying (12) by $P_{D}(x)$ and integrating on $S_{D}$ we obtain

$$
\int_{S_{D}}\left[\mu \cdot f(x)-|\nabla u|^{2}\right] \delta P_{D}(n(x)) d s=0 .
$$

From this it is easy to get

$$
\frac{1}{2} \mu \cdot \int_{S_{D}} f(x) P_{D}(n(x)) d s=\frac{1}{2} \int_{S_{D}}|\nabla u|^{2} P_{D}(n(x)) d s .
$$

As is shown in [7] the right hand side of this equality is equal to the first eigenvalue of the problem (1)-(2)

$$
\lambda_{1}=\frac{1}{2} \int_{S_{D}}|\nabla u|^{2} P_{D}(n(x)) d s
$$

Considering this we get

$$
\mu \cdot \int_{S_{D}} f(x) P_{D}(n(x)) d s=2 \lambda_{1} .
$$

Since the first eigenvalue of the problem (1), (2) is not equal to zero,

$$
\int_{S_{D}} f(x) P_{D}(n(x)) d s \neq 0 .
$$

So we obtain for the Lagrange's multiplier

$$
\mu=\frac{2 \lambda_{1}}{\int_{S_{D}} f(x) P_{D}(n(x)) d s} .
$$

This formula allows one to calculate Lagrange multiplier. Note that here $D$ is an optimal domain and (13) doesn't include an eigenfunction.

Putting (13) into (12) we get 
From this we finally obtain

$$
\frac{2 \lambda_{1} \cdot f(x)}{\int_{S_{D}} f(x) P_{D}(n(x)) d s}=|\nabla u|^{2} .
$$

Suppose, that $f$ is a such function that

$$
\frac{|\nabla u|^{2}}{\lambda_{1}}=\frac{2 f(x)}{\int_{S_{D}} f(x) P_{D}(n(x)) d s} .
$$

$$
\frac{2 f(x)}{\int_{S_{D}} f(x) P_{D}(n(x)) d s}=\varphi(x), x \in S_{D} .
$$

Then for the functions $\varphi(x)=x_{1}^{\alpha} \Phi\left(\frac{x_{2}}{x_{1}}, \frac{x_{3}}{x_{1}}, \ldots, \frac{x_{n}}{x_{1}}\right)$ the solution of $(\mathcal{V})$ problem - the domain $D$ will be a solution of $(\mathcal{I})$ problem at the same time.

There exist two questions here:

a) For which functions $\varphi$ is possible to find functions $f$ providing (16);

b) Investigation of $(\mathcal{V})$ problem.

Now let's investigate the first question.

As is known [8] for the support function $P_{D}(x), x \in E^{n}$ is true

$$
P_{D}(n(x))=(n(x), x) .
$$

Using this and Ostragradskii formulae we can write

$$
\int_{S_{D}} f(x) P_{D}(n(x)) d s=\int_{S_{D}} f(x)(n(x), x) d s=\sum_{i=1}^{n} \int_{D} \frac{\partial}{\partial x_{i}}(f(x), x) d x .
$$

Denoting and calculating the derivatives we can transform

$$
\begin{aligned}
M= & \sum_{i=1}^{n} \int_{D}\left(f(x)+\frac{\partial \cdot f(x)}{\partial x_{i}} x_{i}\right) d x= \\
= & \int_{D}[n \cdot f(x)+(\nabla f, x)] d x,
\end{aligned}
$$

where $\nabla f(x)=\left(\frac{\partial f}{\partial x_{1}}, \ldots, \frac{\partial f}{\partial x_{n}}\right)$.

As follows from condition (7)

$$
M=n+\int_{D}(\nabla f, x) d x
$$

Considering this in (16) we finally obtain

$$
\frac{2 f(x)}{n+\int_{D}(\nabla f, x) d x}=\varphi(x) .
$$

Now we show a class of functions $f$ providing the equivalency of the problems $(\mathcal{I})$ and $(\mathcal{V})$. Take

$$
\varphi(x)=x_{1}^{\alpha} \Phi\left(\frac{x_{2}}{x_{1}}, \frac{x_{3}}{x_{1}}, \ldots, \frac{x_{n}}{x_{1}}\right)
$$


Here $\alpha$ is any given number, $\Phi\left(y_{1}, y_{2}, \ldots, y_{n-1}\right)$ - given differentiable function. We define $f(x)$ as

$$
f(x)=C \cdot x_{1}^{\alpha} \Phi\left(\frac{x_{2}}{x_{1}}, \frac{x_{3}}{x_{1}}, \ldots, \frac{x_{n}}{x_{1}}\right)=C \cdot \varphi(x) .
$$

We can find the constant $C$ from condition (18). It easy to check that for the functions $\varphi(x)$ defined by formula (19) is true

Then from (18) we get

$$
(\nabla \varphi(x), x)=\alpha \varphi(x)
$$

$$
\frac{2 C \varphi(x)}{n+\alpha \int_{D} C \cdot \varphi(x) d x}=\varphi(x) .
$$

Considering here the condition (20) one may obtain

Thus $C=\frac{n+\alpha}{2}$.

$$
2 C \cdot \varphi(x)=(n+\alpha) \varphi(x) .
$$

Other words the function

$$
f(x)=\frac{n+\alpha}{2} \cdot x_{1}^{\alpha} \Phi\left(\frac{x_{2}}{x_{1}}, \frac{x_{3}}{x_{1}}, \ldots, \frac{x_{n}}{x_{1}}\right)=\frac{n+\alpha}{2} \cdot \varphi(x)
$$

satisfies to the condition (18).

Now we give a concrete class of functions satisfying above given conditions. To do it we take the following functions

$$
\varphi(x)=\sum_{i=1}^{n} a_{i} x_{i}^{\alpha} .
$$

In this case the functions $f(x)=\frac{n+\alpha}{2} \varphi(x)$ form the seeking class.

Really, for these functions

Then

$$
f_{x_{i}}=\frac{n+\alpha}{2} a_{i} x_{i}^{\alpha-1}, \quad f_{x_{i}} \cdot x_{i}=\frac{n+\alpha}{2} \alpha a_{i} x_{i}^{\alpha} .
$$

$$
\sum_{i=1}^{n} f_{x_{i}} \cdot x_{i}=(\nabla f, x)=\frac{n+\alpha}{2} \alpha \sum_{i=1}^{n} a_{i} x_{i}^{\alpha}=\alpha \cdot \varphi(x)
$$

consequently

$$
\int_{D}(\nabla f, x) d x=\alpha \int_{D} \sum_{i=1}^{n} \frac{n+\alpha}{2} a_{i} x_{i}^{\alpha} d x=\alpha \int_{D} f(x) d x=\alpha .
$$

If put it into (18) we get

$$
\frac{2 f(x)}{n+\alpha}=\varphi(x) .
$$

That shows that the class was defined correctly.

Now suppose that $\varphi(x) \equiv 1$. Then as a particular case may be obtained from (18)

$$
f(x)=\frac{n}{2} .
$$

So, the condition (4) indeed has a form 


$$
m e s D=\frac{2}{n} .
$$

Other cases also may be considered when the inverse problem (1)-(3) is reduced to the variational formulation (4)-(6) with various functionals. To investigate the problem (4)-(6) one may use the apparatus offered in [6].

Now we consider minimization over domains of some functionals including eigenvalue. As was noted above determination of the minimizing such functionals domains is important for investigation of some inverse problems and the qualitative properties of the eigenvalues.

Consider the problem

$$
\begin{aligned}
& -\Delta u=\lambda u, x \in D \\
& u=0, \quad x \in S_{D} .
\end{aligned}
$$

The second problem is: To find a domain $D \in K$ minimizing the functional

$$
J(D)=\lambda_{1}(D) \cdot \int_{D} f(x) d x,
$$

where $f(x)$ is given in $E^{n}$ function, $D \in K, \lambda_{1}$ is the first eigenvalue of the problem (21)-(22) corresponding to the domain $D$.

If take $f(x) \equiv 1$, then we get the problem

$$
\lambda_{1}(D) \cdot \operatorname{mes} D \rightarrow \min .
$$

As is known, when the domain increases, mesD also increases, but corresponding eigenvalue of this problem decreases. So, it is expectable that this problem has a solution. Also note that this is not true for all functions $f(x)$. We'll give below a class of functions for which this problem has no solution. We also give an optimality condition for the solution of the problem (21)-(22) - the domain $D$, that does not include eigenfunction.

Assume that the problem (21)-(23) has a solution.

Using (9), (10) we can write

$$
\begin{aligned}
\delta J= & \delta \lambda_{1}(D) \cdot \int_{D} f(x) d x+\lambda_{1}(D) \cdot \delta \int_{D} f(x) d x= \\
= & -\int_{S_{D}}|\nabla u|^{2} \delta P_{D}(n(x)) d s \cdot \int_{D} f(x) d x+ \\
& +\lambda_{1}(D) \cdot \int_{S_{D}} f(x) \delta P_{D}(n(x)) d s .
\end{aligned}
$$

From this we get the optimality condition

$$
|\nabla u|^{2} \int_{D} f(x) d x+\lambda_{1}(D) f(x)=0, x \in S_{D} .
$$

Multiplying by $P_{D}$ and integrating over $S_{D}$ we obtain

$$
\left(-\int_{S_{D}}|\nabla u|^{2} P_{D}(n(x)) d s\right) \int_{D} f(x) d x+
$$


From this we get

$$
+\lambda_{1}(D) \int_{S_{D}} f(x) P_{D}(n(x)) d s=0
$$

$$
-2 \lambda_{1} \int_{D} f(x) d x+\lambda_{1}(D) \int_{S_{D}} f(x) P_{D} d s=0 .
$$

Doing above making transformations for $M=\int_{S_{D}}[2 f(x)+(\nabla f, x)] d s$ and considering it in last relation we obtain the optimality condition in the form

$$
\lambda_{1}(D) \cdot \int_{D}(\nabla f, x) d s=0 .
$$

Thus this condition does not include eigenfunction $u(x)$.

Let's consider (25) for some classes of $f$.

a) As a class of $f(x)$ we take the functions satisfying the condition $(\nabla f, x)=\alpha \cdot f(x)$.

Then

$$
\alpha \int_{D} f(x) d x=0 .
$$

Putting it in (24) we get $f(x)=0, x \in S_{D}$. It shows that if $D$ is a solution of the problem (21)-(23) for the functions satisfying a) then

$$
f(x)=0, x \in S_{D}
$$

Indeed the function satisfying a) has a form

$$
f(x)=x_{1}^{\alpha} \Phi\left(\frac{x_{2}}{x_{1}}, \frac{x_{3}}{x_{1}}, \ldots, \frac{x_{n}}{x_{1}}\right)=C \cdot \varphi(x) .
$$

For such functions is true

$$
(\nabla f, x)=\alpha \cdot f(x), x \in D
$$

If we add here the condition

$$
f(x)=0, x \in S_{D}
$$

we get

$$
f(x) \equiv 0, x \in D
$$

So we get the following

Consequence. The problem (21)-(23) has no solution for the functions satisfying (26).

Now let's consider another functional and give a formula for the eigenvalue corresponding to the optimal domain.

Consider the following problem

$$
\begin{gathered}
-\Delta u=\lambda u, \quad x \in D, \\
u(x)=0, \quad x \in S_{D},
\end{gathered}
$$

where $D \in K$.

The third problem is: To find $D \subset K$, such that 


$$
J(D)=\lambda_{1}(D)+\int_{D} f(x) d x \rightarrow \min ,
$$

where $\lambda_{1}(D)$ is the first eigenvalue of the problem $(27),(28)$ corresponding to $D \subset K, f(x)$ given continuously differentiable in $E^{n}$ function.

We suppose that the problem (27)-(29) has a solution on $K$.

Theorem. If the domain $D \in K$ is a solution of the problem (27)-(29), then

$$
\lambda_{1}(D)=\frac{1}{2} \int_{S_{D}} f(x) P_{D}(n(x)) d s .
$$

Proof. Considering (9) we can write

$$
\delta \lambda_{1}(D)=-\int_{S_{D}}\left|\nabla u_{1}(x)\right|^{2}\left[P_{D^{\prime}}(n(x))-P_{D}(n(x))\right] d s,
$$

where $D, D^{\prime} \in K$.

Let's take positive parameter $t$ and define $D=D(t), \lambda_{1}(t)=\lambda_{1}(D(t))$. Then as one may obtain from (30)

$$
\begin{gathered}
\lambda_{1}(t+\Delta t)-\lambda_{1}(t)=\lambda_{1}(D(t+\Delta t))-\lambda_{1}(D(t))= \\
=\int_{S(t)}\left|\nabla u_{1}(x)\right|^{2}\left[P_{D(t+\Delta t)}(n(x))-P_{D(t)}(n(x))\right] d s+o(\Delta t) .
\end{gathered}
$$

From this dividing by $\Delta t$ we get

$$
\lambda_{1}^{\prime}(t)=-\int_{S_{D(t)}}\left|\nabla u_{1}(x)\right|^{2} P_{D(t)}^{\prime}(n(x)) d s,
$$

where

$$
P_{D(t)}^{\prime}(x)=\frac{d}{d t} P_{D(t)}(x)
$$

Now let's show that for the first eigenvalue of the problem $(27),(28)$ in the domain $D$ is true the formula

$$
\lambda_{1}(D)=\frac{1}{2} \int_{S_{D}}\left|\nabla u_{1}(x)\right|^{2} P_{D}(n(x)) d s .
$$

Here we denoted by $u_{1}(t)$ the first eigenfunction of the problem (27), (28) corresponding to the domain $D_{0}$. Take $D_{0} \in K, D(t)=t D_{0}, \quad t>0$. Then it is clear that the function $\tilde{u}_{1}(x)=u_{1}\left(\frac{x}{t}\right), x \in D(t)$ would be an eigenfunction of the problem $(27),(28)$ by $D=D(t)$. Really, considering

$$
\Delta \tilde{u}_{1}(x)=\frac{1}{t^{2}} \Delta u_{1}\left(\frac{x}{t}\right)
$$

we see that $\tilde{u}_{1}(x)$ is an eigenfunction of $(27),(28)$ corresponding for the eigenvalue $\lambda_{1}(t)=$ $\frac{\lambda_{1}\left(D_{0}\right)}{t^{2}}$. Considering this in (31) we have

$$
-2 \frac{\lambda_{1}\left(D_{0}\right)}{t^{3}}=-\frac{1}{t^{2}} \int_{S_{D}}\left|\nabla u_{1}\left(\frac{x}{t}\right)\right|^{2} P_{D_{0}}(n(x)) d s .
$$


Taking $t=1$ we obtain (32).

Considering (10) for the functional

$$
F(D)=\int_{D} f(x) d x
$$

we have

$$
\delta F(D)=\int_{S_{D}} f(x)\left[P_{D^{\prime}}(n(x))-P_{D}(n(x))\right] d s .
$$

Now, let $D \in K$ be solution of the problem (27)-(29). Then according to the optimality condition

$$
-\left|\nabla u_{1}(x)\right|^{2}+f(x)=0, \quad x \in S_{D} .
$$

Multiplying (33) by $P_{D}(n(x))$ and integrating over $S_{D}$ we get

$$
-\frac{1}{2} \int_{S_{D}}\left|\nabla u_{1}(x)\right|^{2} P_{D}(n(x)) d s+\frac{1}{2} \int_{S_{D}} f(x) P_{D}(n(x)) d s=0 .
$$

Considering here (32) we get the statement of the theorem.

Theorem is proved.

Consider some particular cases. Suppose that $f(x)=1, x \in E^{n}$. In this case the functional (29) takes a form

$$
J(D)=\lambda_{1}(D)+\text { mes } D \rightarrow \text { min } .
$$

From the theorem we obtain

$$
\lambda_{1}(D)=\frac{1}{2} \int_{S_{D}} P_{D}(n(x)) d s .
$$

In two dimensional case $([6])$

$$
\frac{1}{2} \int_{S_{D}} P_{D}(n(x)) d s=m e s D .
$$

Thus

$$
\lambda_{1}(D)=\text { mes } D .
$$

In one dimensional case the problem (27)-(29) has the form

$$
\begin{aligned}
& u^{\prime \prime}=\lambda u, x \in(a, b), \\
& u(a)=u(b)=0, \\
& \lambda_{1}(a, b)+\int_{a}^{b} f(x) d x \rightarrow \min .
\end{aligned}
$$

In this case finding of domain is equivalent to the finding of $a$ and $b$. For this problem

$$
\lambda_{1}(a, b)=\frac{1}{2}[f(b) b-f(a) a] .
$$

For considered problem the eigenvalues are determined (see for instance [3]), so it is not difficult to check the obtained formulae. 
Note, that when $f(x) \equiv 1$ the problem (27)-(29) has no solution. In this case the "increasing" of domain leads to decreasing of the eigenvalue. From (32) also follows the condition $\lambda_{1}=0$, that is not true for considered problem.

Using the offered here technique other cases also may be considered.

Authors wish to thank to prof. A.Nachaoui for discussion and useful remarks.

Acknowledgements. This work has been done during a 'invited professor' visit of Y.S.Gasimov in Universite de Nantes. The author wish to thank to the administration of the university for the invitation and the Laboratory de Mathematique for the kind and warm hospitablity.

\section{References}

1. S.H.Gould. Variational Methods for Eigenvalue Problems. Univ.of Toronto Press. London: Oxford Univ. Press, 1996.

2.D.Bugur, G.Buttazzo, I.Figueiredo. On the Attainable Eigenvalues of the Laplace Operator. SIAM J.Math.Anal., 30 (1999) , 527-536.

3. V.S.Vladimirov. Equations of Mathematical Physics. M.: Nauka 1988.

4. V.P.Mikhailov. Partial Differential Equations.M.: Nauka,1976.

5. Y.S.Gasimov, A.A.Niftiev. On a Minimization of the Eigenvalues of Shrodinger Operator over Domains. Doclady RAS, 2001, v.380, 3, p.305-307.

6. A.A.Niftiyev,Y.S.Gasimov. Control by Boundaries and Eigenvalue Problems with Variable Domains. Publishing House of Baku State University, 2004.

7. Y.S.Gasimov. On Some Properties of the Eigenvalues when the Domain Varies. Mathematical Physics, Analyses and Geometry. 2003, v. 10, N2, p.249-255.

8. V.F.Demyanov, A.M.Rubinov. Basises of Non- Smooth Analyses and Quazidifferential Calculas. Nauka, Moskow, 1990.

8. 